\section{Mejora de la calidad aplicando la metodologia de superficie respuesta y redes neuronales}

\author{
Recepción: Mayo de $2006 /$ Aceptación: Junio de 2006
}

(1) Javier Del Carpio Gallegos

(2) Juan Cevallos Ampuero

(3) Roberto Eyzaguirre Tejada

(4) Rosmeri Mayta Huatuco

(5) Edagra Ruiz Lizama

(6) Julio Yenque Dedios

\section{RESUMEN \\ El artículo presenta un resumen del proyecto de investigacion realizado por el responsable y los miembros del proyecto de investigacion, y muestra las ventajas del uso de redes neuronales frente a otras técnicas sobre mejora de la calidad, llegando a Cuadrados Medios de Error más bajos(MSE). \\ Palabras Clave: Calidad, superficie respuesta, redes neuronales. \\ IMPROVE QUALITY APPLING METHODOLOGY ANSWER SURFACE AND NEURAL NETWORKS ABSTRACT \\ This article show an abstract of research proyect doing by the manager y collaborators of proyect, show the advantages of use neural networks opposite another techniques about improve quality, to get mean error squares more lows (MSE).}

Key words: Quality, answer surface, neural network.

\section{INTRODUCCIÓN}

El objetivo del proyecto fue desarrollar una metodología para mejorar la calidad de procesos, productos y/o servicios, mediante el uso de MSR Metodología de Superficie de Respuesta y RN Redes Neuronales.

La mejora de la calidad en la actualidad se puede realizar en la línea y fuera de la línea; en los casos de fuera de la línea por lo general se usan los diseños de experimentos; sin embargo ellos tienen la limitación que trabajan con relaciones lineales, lo cual no siempre es lo más adecuado; por ello se busca una metodología de mejora de la calidad que trabaje con relaciones no lineales y que sea confiable.

Para conseguir el objetivo se requiere analizar información sobre aplicación de MSR Metodología de Superficie de Respuesta para mejorar la calidad, seleccionar una metodologia que sea práctica de usar para este objetivo de la calidad.

Adicionalmete, se requieren seleccionar las $\mathrm{RN}$ específicas más adecua das para el objetivo de la calidad, considerando que pueden ser recurrentes y no recurrentes. $Y$ dentro de ello existen diversas redes alternativas.

Se presentará un caso estudio en el cual se evidencie la aplicación de las metodología MSR y RN, mostrando la mejora en los indicadores de calidad.

\section{METTODOS Y RESULTADOS}

\section{Hipótesis}

Es posible desarrollar una metodología de mejora de la calidad mediante el uso combinado de MSR Metodología de Superficie de Respuesta y RN Redes Neuronales.

\section{Ventajas}

La gran ventaja del uso de las redes neuronales frente a las demás metodologías de uso tradicional es que pueden trabajar con relaciones lineales y no lineales. Sin embargo, se presenta la limitación de que muchas veces se encuentran óptimos locales y no generales, ello se puede

(1) Magíster en Ciencias. Profesor del Departamento de Producción y Gestión Industrial, UNMSM. E-mail: jdelcarpio1@yahoo.es

(2) Magíster en Ingeniería Industrial. Profesor del Departamento de Producción y Gestión Industrial, UNMSM. E-mail: jcevallosa@unmsm.edu.pe

(3) Ingeniero Industrial. Profesor del Departamento de Ingeniería de Sistemas e Informática, UNMSM. E-mail: r_eyzaguirre@viabcp.com

(4) Ingeniero Industrial. Profesor del Departamento de Ingeniería de Sistemas e Informática, UNMSM. E-mail: rmaytah@unmsm.edu.pe

(5) Ingeniero Industrial. Profesor del Departamento de Ingeniería de Sistemas e Informática, UNMSM E-mail: eruizl@unmsm.edu.pe

(6) Ingeniero Industrial. Profesor del Departamento de Diseño y Tecnología Industrial, UNMSM. E-mail: jyenqued@unmsm.edu.pe 
〉> Javier Del Carpio G., Juan Cevallos A., Roberto Eyzaguirre T., Rosmeri Mayta H., Edgar Ruiz L. y Julio Yenque D.

superar usando otras técnicas que nos permitan realizar la aproximación gruesa, para ello se propone usar la metodología de superficie de respuesta, la misma que nos va a permitir obtener un primer resultado, que será optimizado con la aplicación de redes neuronales

\section{Metodología}

El diseño experimental será con base al análisis factorial.

Primero, se recolectará información sobre MSR y RN. Luego, se analizará la información y se desarrollará una metodología sobre mejora de la calidad.

Se aplicará la metodología en productos específicos elaborados en el Perú, con base a ello se probará la metodología. Asimismo, se aplicará diseño de experimentos a los diseños experimentales seleccionados, con base a sus resultados se analizará los mismos, seguidamente se aplicarán redes neuronales, haciendo un análisis de las diversas alternativas de redes neuronales.

Posteriormente, se seleccionarán las arquitecturas más convenientes, y se tomarán en cuenta redes recurrentes y no recurrentes. Además, se compararán los resultados tradicionales con los obtenidos con redes neuronales.

Luego se analizarán los resultados obtenidos aplicando redes neuronales y los que se obtengan aplicando metodología de supérficie de respuestas previa a la aplicación de redes neuronales.

Se simularán procesos de elaboración de productos y en ellos se aplicarán las diversas metodologías tradicionales y las de redes neuronales con metodología de superficie de respuesta comparándose y analizando los pro y contra de ambas alternativas.

El enfoque propuesto para respuesta de calidad de manera cualitativa está dado por lo siguiente:

1. Establecer los conjuntos de entrenamiento y de prueba de la red uno. Tomar al azar $20 \%$ de los datos experimentales para formar el conjunto de prueba y los restantes forman el conjunto de entrenamiento.

2. Construir la arquitectura de la red uno y determinar el conjunto de parámetros óptimos para los factores controlables.

a. Calcular las probabilidades acumuladas para todas las respuestas cualitativas (en cada experimento, para cada nivel).

b. Construir la red uno, asignando la probabilidad acumulada de cada categoría de la respuesta cualitativa como el input; los correspondien- tes conjuntos de valores de los factores controlables son asignados como el output. Probar diversas arquitecturas de red backpropagation mediante el uso del conjunto de entrenamiento y prueba seleccionados en el paso 1 (arquitectura: número de neuronas en la capa de entrada, capa oculta y capa de salida). El error cuadrado medio MSE de cada arquitectura puede ser utilizada como el criterio para determinar la mejor arquitectura. Un número predeterminado de ciclos de entrenamiento puede ser considerado como el criterio para detener el proceso de entrenamiento. La mejor arquitectura es aquella que puede simultáneamente minimizar el MSE del conjunto de entrenamiento y de la prueba del paso 1.

c. Ingrese las probabilidades acumuladas deseadas como input de la red uno encontrada en b) y calcule los output respectivos, que en este caso serán los factores controlables óptimos.

3. Construir la arquitectura de la red dos para predecir las probabilidades acumuladas de las respuestas cualitativas.

a. Asignar el conjunto de valores de factores controlables de 1, como el input de la red dos. La correspondiente probabilidad acumulada de cada clase es asignada como la salida de la red dos.

b. Construir la red dos, probando varias arquitecturas de la red dos (número de neuronas en las capas de entrada, oculta y de salida) mediante la combinación de los conjuntos de entrenamiento y prueba de 1.

c. Ingrese el conjunto óptimo de parámetros encontrados en 2, para la mejor red backpropagation encontrada en (b). La probabilidad acumulada predicha de cada categoría puede ser entonces obtenida.

4. Comparar el resultado estimado con el objetivo. Si el resultado estimado en el paso 3.c no se aparta de manera significativa del valor objetivo, el análisis se ha completado. Si no fuera así, retorne al paso 1 para reformar el conjunto de entrenamiento y prueba y repita los pasos 2 a 4 hasta que la desviación entre los resultados estimados y el valor objetivo sea aceptable.

\section{A P L I CACIÓN \\ Descripción del problema y característica de calidad a obtener \\ Se estudió un proceso de elaboración de caramelos. La característica de calidad que se buscaba es que no quede meloso a la semana de estar a la intemperie.}


Cuadro 1. Definición cualitativa de las clases

\begin{tabular}{|l|l|}
\hline Categoría & Definición \\
\hline I. La menor melosidad & $\begin{array}{l}\text { Al tacto no queda nada pegajoso en el dedo y no } \\
\text { se aprecia variación sobre la superficie. }\end{array}$ \\
\hline II Melosidad aceptable & $\begin{array}{l}\text { Al tacto los dedos quedan ligeramente pegajosos, } \\
\text { pero visualmente no se aprecia variación sobre la } \\
\text { superficie. }\end{array}$ \\
\hline III La peor melosidad & $\begin{array}{l}\text { Al tacto los dedos quedan pegajosos, y visualmente } \\
\text { se aprecia variación sobre la superficie. }\end{array}$ \\
\hline
\end{tabular}

La melosidad es juzgada por inspección al tacto de los ingenieros de procesos. Mediante un número indicativo del nivel de adhesión de los dedos al estar en contacto con el caramelo es la forma de evaluación.

El nivel de melosidad puede ser clasificado en varias clases de acuerdo al nivel de adhesión que se produce al tacto. Los ingenieros de procesos determinaron 3 clases: la menor melosidad I, melosidad aceptable II, la peor melosidad - (se derrite) III.

El cuadro 1 precisa la definición cualitativa de tres clases para la melosidad.

Reconocimiento del estado actual En planta los ingenieros escogen series pequeñas fuera de la línea para la inspección. Las pequeñas series fuera de las líneas seleccionadas son de 24 caramelos, los mismos que son evaluados mediante una inspección simple.

El estado actual de las probabilidades acumuladas, respectivamente, para las 3 clases I, II, III revela: $0.64000,0.7520,1.000$. Los resultados ideales de probabilidades acumuladas de las 3 clases son: $1.0000,1.0000,1.0000$. Obviamente, el resultado actual está lejos del resultado ideal. Para mejorar la característica de la melosidad, es necesario optimizar parámetros.
Determinación experimental de factores y diseño experimental

Hay varias variables del proceso que pueden afectar la calidad de la melosidad que se produce en la superficie después del proceso. Luego de analizar los mecanismos relacionados mediante un trabajo de tormenta de ideas, los ingenieros de procesos seleccionaron cuatro variables del proceso: la cantidad de azúcar que se agrega por lote, la temperatura de proceso, el tiempo de proceso y el tiempo de mezclado para obtener la masa caramelo. El cuadro 2 precisa los cuatro factores y sus definiciones de niveles. Para reducir el tiempo y costo experimental se selecciona un arreglo ortogonal L9, de los modelos de diseños experimentales que se proponen en los Métodos Taguchi (10). Cada prueba experimental se repitió 6 veces.

Resultados del análisis mediante la aplicación del enfoque propuesto

El $20 \%$ de lo datos experimentales se seleccionan al azar para formar parte del conjunto de prueba y el resto es usado para el conjunto de entrenamiento (este ejemplo incluye 54 datos experimentales). Se utiliza el software Matlab para desarrollar las redes requeridas. Para obtener el conjunto de valores óptimos del paso 2, se utilizan las probabilidades acumuladas de cada clase para cada dato que fueron calculadas inicialmente. Luego las probabilidades acumuladas de los valores de las 3 clases de los

Cuadro 2. Definición de niveles de factores controlables

\begin{tabular}{|l|c|c|c|}
\hline Factor & Nivel 1 & Nivel 2 & Nivel 3 \\
\hline \begin{tabular}{l} 
A. $\begin{array}{l}\text { Cantidad de azúcar que se } \\
\text { agrega por lote, } \mathrm{kg}\end{array}$ \\
\hline B. Temperatura de proceso. ${ }^{\circ} \mathrm{C}$
\end{tabular} & 75 & 12 & 14 \\
\hline C. Tiempo de proceso, min. & 30 & 35 & 85 \\
\hline $\begin{array}{l}\text { D. Tiempo de mezclado para } \\
\text { obtener la masa caramelo, min }\end{array}$ & 20 & $25^{*}$ & 30 \\
\hline
\end{tabular}

* Valor inicial establecido para el factor controlable. 


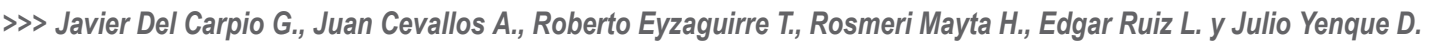

Cuadro 3 . Resultados de confirmación de experimentos para establecer los parámetros óptimos

\begin{tabular}{|c|c|c|c|c|c|c|c|c|c|c|}
\hline No. & \multicolumn{4}{|c|}{$\begin{array}{c}\text { Parámetros } \\
\text { establecidos }\end{array}$} & \multicolumn{4}{c|}{ La cantidad de cada clase } & \multicolumn{3}{|c}{$\begin{array}{c}\text { Las probabilidades } \\
\text { acumuladas de cada } \\
\text { clase }\end{array}$} \\
\hline & A & B & C & D & I & II & III & (I) & (II) & (III) \\
\hline 1 & 10.2 & 79.0 & 36.1 & 19.6 & 22 & 2 & 0 & 0.9167 & 1.000 & 1.000 \\
\hline 2 & 10.2 & 79.0 & 36.1 & 19.6 & 21 & 2 & 1 & 0.8750 & 0.9583 & 1.000 \\
\hline 3 & 10.2 & 79.0 & 36.1 & 19.6 & 23 & 1 & 0 & 0.9583 & 1.000 & 1.000 \\
\hline \multicolumn{2}{|c|}{ Resultados promedios } & 22 & 1.667 & 0.333 & 0.9167 & 0.9861 & 1.000 \\
\hline
\end{tabular}

(I), (II), (III) significan las probabilidades acumuladas de las clases I, II, III, respectivamente.

factores son entonces considerados como entradas de la red backpropagation 1. En este caso se eligió la realización de 2000 ciclos como criterio de detención de un proceso de entrenamiento. Se encontró que la estructura 3-12- 4 (número de neuronas en las capas interna, oculta y externa, respectivamente) es seleccionada por tener el mejor desempeño (entrenamiento MSE $=0.0573$ y prueba MSE $=0.0987$ ) con 2000 ciclos de entrenamiento.

Luego, debe introducir las probabilidades acumuladas deseadas $(1.000,1.000,1.000)$ de las respuestas de las categorías cualitativas para la red backpropagation 1 , los valores óptimos establecidos de los factores controlables obtenidos son: la cantidad de azúcar que se agrega por lote $=10.174342 \mathrm{~kg}$, la temperatura de proceso $=79.047798^{\circ} \mathrm{C}$, el tiempo de proceso $=$ 36.110775 min.y el tiempo de mezclado para obtener la masa caramelo $=19.625937 \mathrm{~min}$.

A continuación, para obtener la probabilidad acumulada para cada categoría en el paso 3, se construye la red backpropagation 2 asignando el conjunto de valores de los factores controlables y de las probabilidades acumuladas de las 3 clases para ser las entradas y salidas de la red backpropagation 2. La estructura obtenida fue 4-9-3 por tener el mejor desempeño (entrenamiento MSE $=0.045286$ y prueba MSE $=0.0652$ ). Las probabilidades acumuladas estimadas de las 3 clases son obtenidas ingresando los valores establecidos como parámetros óptimos, obtenidos en el paso 2, en la red back propagation 1; los mismos que son: $10.174342 \mathrm{Kg} ., 79.047798^{\circ} \mathrm{C}$, 36.110775 min., 19.625937 min., respectivamente. Las probabilidades acumuladas estimadas para las 3 clases del proceso de elaboración del caramelo son: $0.779073,0.877350,0.961793$. Los valores estimados están cerca de la calidad deseada de: 1.0000 , $1.0000,1.0000$, por lo tanto, la efectividad del enfoque propuesta debería ser confirmada.

El conjunto de valores óptimos continuos establecidos para los factores controlables pueden ser obteni- dos mediante el empleo del enfoque propuesto, hay algunas dificultades para establecer los valores en la práctica. Después de discutir con los ingenieros de proceso los valores se establecen fácilmente, los cuáles están cerca del conjunto de parámetros óptimos estimados que son determinados como: 10.2 Kg., $79.0^{\circ} \mathrm{C}, 36.1 \mathrm{~min}$., $19.6 \mathrm{~min}$., respectivamente. Finalmente, un experimento confirmatorio con 3 repeticiones se realizó para verificar los resultados. La tabla 3 precisa los resultados de las 3 repeticiones. Las probabilidades acumuladas promedio de las 3 clases puede ser encontrada como $(0.9167,0.9861$, $1.000)$. El resultado confirmado de $(0.9167,0.9861$, $1.000)$ está cerca del resultado estimado de $(0.779073,0.877350,0.961793)$.

Por lo tanto los ingenieros de proceso pueden aceptar el conjunto de valores que hemos encontrado. Además, el resultado confirmado $(0.9167,0.9861$, 1.000) efectivamente logra una mejor calidad que el resultado presente de $(0.6400,0.7520,1.000)$ en el proceso de elaboración del caramelo. La eficacia del enfoque propuesto puede ser verificada mediante la demostración de este caso.

\section{ANALISIS Y DISCUSIÓN}

En la actualidad en muchos casos dada la complejidad de los productos manufacturados, puede ser justificado calcular una respuesta cuantitativa. Asimismo, la tendencia a emplear evaluaciones cualitativas, está aumentando gradualmente.

Es así que, este estudio propone un nuevo enfoque basado en la técnica de redes neuronales para lograr los parámetros de optimización para respuestas de calidad con categorías de orden cualitativo.

Después de obtener los parámetros de optimización necesarios, una mejora en la calidad puede ser lograda. Y el caso demuestra la efectividad del enfoque propuesto. 
El uso de las redes neuronales ha permitido un error menor en los resultados y es necesario en el caso que se trabaje en partes por millón de defectuosos, lo cual es justamente uno de los mayores retos actuales de la mejora de la calidad a nivel internacional.

Por otro lado es de destacar que el uso de metodología de superficie de respuesta permite resultados más preciso ya que permite afinar la selección de variables independientes, y los parámetros respectivos son determinados con más excatitud.

Con relación al tipo de red neuronal, es de destacar la ventaja de usar redes neuronales supervisadas, y dentro de ello el uso del algoritmo backpropagation. El uso de redes que cuentan con una capa oculta es lo que mejores resultados ha dado.

\section{CONCLUSIONES}

Se tiene al alcance una metodología de mejora de la calidad que permita una efectiva reducción de costos y aumento de eficiencia.

En la actualidad las técnicas de mejora de la calidad son fundamentalmente cualitativas y pocas cuantitativas. En el caso de las cuantitativas, la mayoría trabaja con el supuesto de relaciones lineales lo cual es una limitante por cuanto la realidad no es lineal sino todo lo contrario.

En lo que respecta a metodologías de trabajo fuera de la línea, son pocas las alternativas. Por lo tanto, contar con una metodología de mejora de la calidad fuera de la línea permitirá lograr mejores niveles de calidad en actividades de diseño y producción.

\section{REFERENCIAS BIBLIOGRÀFICAS}

1. April, J, Glover, F, Kelly, J, Laguna, M. Practical Introduction to simulation optimization. Proceedings of the 2003 Winter Simulation Conference. 2003.

2. Del Rio y Sanz. Redes Neuronales y Sistemas Difusos, Editorial Alfaomega.2002.
3. Glover, F, Kelly, J, Laguna, M. New advances and applications of combining simulation and optimization. Proceedings of the 1996 Winter Simulation Conference. 1996.

4. Hagan, M., et al. Matlab Guide of tools. 2001.

5. Hilera y Martínez. Redes Neuronales Artificiales, Editorial Alfaomega.2000.

6. Hsieh, K y Tong, L. Parameter optimization for quality response with linguistic ordered category by employing artificial neural networks: a case study. Integrated Manufactured Systems. 2000.

7. Hsieh, K. Process improvement I the presence of quality response by combining fuzzy sets and neural networks. Integrated Manufactured Systems. 2001.

8. Jean, Y.C. Quality Improvement for RC 06 Chip Resistor. Quality and Reliability Engineering International. Vol. 12. 1996.

9. Journal of Food Science. IFT. Editorial IFT.

10.Kaufmann Arnold, Grafos Neuronales para la Economía y la Gestión de Empresas. Ed. Pirámide.1995.

11.Konak, A., Jonak, S., Smith, A, Nettleship, J. Estimation of shrinkage for near - shape using a neural network approach. Journal of Intelligent Manufacturing. Apr.2003.

12. Martin Del Brio, B y Sanz, A. Redes neuronales y Sistemas Difusos. Editorial Alfaomega. 2002.

13.Montgomery, Diseño y Análisis de Experimentos. Editorial Iberoamerica. 2004.

14.Nair, V.N. Testing in Industrial Experiments with ordered categorical data. Technometrics. Vol 28. 1986.

15.Taguchi, G. Quality Engineering. Mc GrawHill.1986. 